\title{
ESTIMACIÓN DEL COMPORTAMIENTO FUTURO DEL NIVEL DEL MAR EN LAS ISLAS CANARIAS A PARTIR DEL ANÁLISIS DE REGISTROS RECIENTES
}

\author{
P. Fraile Jurado, E. Sánchez Rodríguez, \\ M. Fernández Díaz y M.F. Pita López \\ Departamento de Geografía Física y A.G.R. Universidad de Sevilla \\ C/ María de Padilla s/n. CP 41004, Sevilla \\ pfraile@us.es \\ J.M. López Torres \\ Departamento de Geografía Humana. Universidad de Sevilla \\ C/ María de Padilla s/n. CP 41004, Sevilla
}

\begin{abstract}
Resumen: En este artículo se estima el comportamiento futuro del nivel medio del mar en las Islas Canarias a partir de registros locales procedentes de mareógrafos y satélites altimétricos (con información sobre el comportamiento reciente del nivel del mar en la superficie marina). La metodología empleada consiste en realizar un análisis de regresión lineal entre dichos registros locales recientes y una serie global correspondiente al mismo periodo temporal, identificando en cada una de las rectas de regresión lineal correspondiente los valores referidos a tres escenarios futuros del IPCC, que indican las expectativas locales de cambio del nivel del mar tanto en los mareógrafos como en la superficie marina. Los resultados obtenidos muestran que se producirán subidas generalizadas del nivel medio del mar en todo el área de estudio. En dos de los mareógrafos analizados y en la mayor parte de las aguas canarias se producirán subidas por encima de las expectativas del IPCC, mientras que en el tercer mareógrafo, en Lanzarote, los resultados indican ascensos del nivel del mar inferiores a las predicciones globales para el final del siglo XXI.
\end{abstract}

Palabras clave: subida del nivel del mar, mareógrafo, altimetría por satélite, regresión lineal, Islas Canarias.

Recibido: 10-9-14. Aceptado: 3-12-14. 


\begin{abstract}
The aim of this paper is to estimate the future mean sea level rise in the Canary Islands by means of the analysis of tide gauge and altimetry satellite records. The applied methodology consists of a linear regression analysis between global and local time series sea level rise data over the same time period. An equation of the linear regression line is obtained for each pair of data in the time series (global and local), corresponding values for three IPCC scenarios in the global variable are identified, and a local prediction is obtained. A higher sea level rise than IPCC predictions have been found in two of the analyzed tide gauges and in most of the studied water surface. Obtained results in Lanzarote show lower sea level rises than IPCC predictions by the end of the 21st century.
\end{abstract}

Keywords: mean sea level rise, tide gauge, satellite altimetry, linear regression analysis, Canary Islands.

\title{
1. Introducción
}

El nivel medio del mar es una variable esencial para comprender el comportamiento de numerosos procesos físicos. Los cambios pasados del nivel del mar se han empleado como variable principal a la hora de explicar la erosión costera (Bruun, 1954) o la intensidad y frecuencia de las inundaciones causadas por temporales (Pugh, 1996), dedicándose numerosos estudios a registrar el comportamiento interanual de esta variable (Gornitz et al., 1982, Douglas et al., 2000).

Asimismo, la subida del nivel del mar es uno de los principales retos a los que nos enfrentaremos como sociedad durante el siglo XXI (Stocker et al., 2013). De acuerdo con el IPCC (IPCC, 2007), existe una necesidad de estudios sobre el comportamiento futuro del nivel medio del mar a escala local para la planificación urbana y territorial. No obstante, raramente se cuenta con este tipo de información para la elaboración de planes de ordenación del territorio, debido principalmente a que las fuentes de información sobre el comportamiento local del nivel medio del mar suelen ser discontinuas espacial y temporalmente, tal y como es el caso de la red de mareógrafos españoles (Puertos del Estado, 2009).

La subida del nivel medio del mar es una de las consecuencias del cambio climático que menos se ha puesto en duda. Pese a que en la década de los 70 y principios de los 80 determinados trabajos identificaron evidencias de un posible descenso del mismo a escala global (Rice et al., 1982), a partir de los 80 y sobre todo a principios de los 90 diferentes autores mencionan la existencia de un proceso global de ascenso del nivel del mar (Woodworth, 1999, Dean et al., 1987, Douglas et al., 2000), relacionándolo en la mayoría de los casos con el proceso de calentamiento global. Por lo tanto, 
y a diferencia de otras áreas vinculadas al cambio climático en las que hasta hace pocos años se ha discutido sobre su ocurrencia o no (el propio fenómeno de calentamiento global, sus causas, el incremento de fenómenos meteorológicos extremos) la mayor parte de la discusión científica sobre los cambios del nivel medio del mar se han centrado principalmente en 3 líneas:

- La cuantificación de los cambios globales ocurridos hasta el presente. La mayor parte de los trabajos coinciden en identificar un fenómeno de aceleración en las tasas de subida del nivel del mar de carácter eustático, pasando de un promedio de unos 1.7 mm/año en todo el siglo XX a 3.4 desde 1992 (Moss, 2011, Camino et al., 2014). Estos últimos datos se pueden observar tanto en los registros de los mareógrafos como en los de los satélites altimétricos, tras unos años de ligeras diferencias entre ambos explicadas por la escasa duración de las series temporales de estos últimos (Ablain et al., 2009). No obstante, diferentes autores han llamado la atención sobre la gran variabilidad espacial del fenómeno de cambio del nivel del mar (Church y White, 2011). En el período 1992-2013 se observan promedios que oscilan entre -2 y $+10 \mathrm{~mm} /$ año en función del área marina objeto de estudio.

- La realización de predicciones sobre expectativas globales futuras. Esta es la cuestión que ha concitado mayores desavenencias. Durante la década de los 90 se produjo una evolución a la baja en las expectativas de subida del nivel medio del mar, desde las subidas esperadas de más de 4 metros para el año 2100 (Hoffman et al., 1983) hasta poco más de 0.5 metros en el informe del IPCC de 2007 (Solomon, 2007). Desde el informe de 2007 se ha observado un ligero repunte en las expectativas de cambio del nivel del mar. Esta modificación está asociada tanto al incremento en la complejidad de los modelos de cambio climático empleados como a los sucesivos cambios en los escenarios de emisiones de gases de efecto invernadero durante el resto del siglo XXI. Actualmente permanece en discusión la propuesta de varios autores (Rahmstorf, 2007, Pfeffer et al., 2008), que modificaron significativamente al alza las predicciones del informe del IPCC de 2007 tras un reanálisis de los registros recientes de cambios del nivel del mar, sin que en el informe del IPCC de 2013 se haya llegado a un consenso entre los defensores de este tipo de análisis y los autores de los modelos de cambio climático (IPCC, 2013).

- La elaboración de estudios de carácter regional y local, generalmente vinculados al análisis de datos medidos en los siglos XX y XXI. Se han realizado numerosas publicaciones sobre la evolución pasada del nivel medio del mar registrada tanto en mareógrafos como a través de los datos procedentes de altimetría por satélite. No obstante, en el caso español, este tipo de trabajos son escasos, y están mayoritariamente centrados en el análisis de las series temporales de los mareógrafos peninsulares (Tel y García, 2002, Marcos et al., 2005). La aproximación cuaternarista, basada en el análisis de los registros de posiciones altas del nivel del mar (estadios isotópicos 11 y 5e, principalmente) ha sido significativamente más 
frecuente tanto en la literatura científica internacional (Lambeck et al., 2011) como nacional (Zazo et al., 1996, Zazo et al., 2005). En general son pocas las publicaciones que tratan de realizar predicciones sobre el futuro del nivel medio del mar a partir del análisis de registros locales recientes, destacando el trabajo de Titus y Narayanan (1995) como una de las escasas aproximaciones analíticas a esta cuestión. En España resultan significativamente menos frecuentes los estudios sobre la evolución futura del comportamiento del nivel medio del mar en áreas concretas (Pardo et al., 1991, Fraile y Ojeda, 2012).

Este artículo se centra en este último aspecto, el análisis de registros recientes del nivel del mar, con la intención de realizar predicciones sobre el futuro a escala local. En los trabajos sobre este tema, la estimación de expectativas locales sobre una futura subida del nivel del mar se ha afrontado desde diferentes aproximaciones metodológicas:

i) La aproximación más sencilla consiste en usar escenarios globales de subida del nivel medio del mar para estimar inundaciones locales futuras, sin tener en cuenta ningún tipo de registro local y asumiendo, por tanto, que no existirá variabilidad espacial en este fenómeno en el futuro (Medina et al., 2004 Hallegate et al., 2011, Timmerman et al., 2010), o bien que dicha variabilidad será mínima (Titus y Richmann, 2001).

ii) La asunción de que la subida del nivel medio del mar registrada por satélites altimétricos no ha sido ocasionada por el calentamiento global, sino por factores exclusivamente locales (por ejemplo, isostáticos), lo que lleva a desarrollar escenarios futuros en los que simplemente se añaden las proyecciones de subida del nivel medio del mar a las alturas locales del nivel medio del mar. Esta aproximación lleva a contar doblemente cualquier parte de la tendencia histórica causada por movimientos eustáticos (Titus y Narayanan, 1995).

iii) La asunción de que cualquier cambio en el nivel del mar registrado en un lugar determinado contiene contribuciones tanto de una parte global como local, ya sea esta última causada por movimientos locales de la superficie marina $\mathrm{o}$, en el caso de tratarse de registros de un mareógrafo, de movimientos verticales de la superficie emergida sobre la que el mareógrafo esté situado (Woodworth et al., 1999). Para poder realizar predicciones sobre futuros niveles del mar, es preciso descomponer las tendencias observadas en las componentes global (eustasia, estimada entre 12 y $18 \mathrm{~cm}$ durante el siglo XX) y local (isostasia). De este modo es posible sumar las expectativas globales procedentes de escenarios como los del IPCC con extrapolaciones de la componente local, evitando así sumar doblemente la tendencia histórica.

En décadas pasadas la importancia de la aproximación elegida fue menor, ya que en la mayor parte de las costas su valoración sólo implica modificaciones de rango submétrico, que resultaban despreciables frente a los escenarios empleados en los 80 y 90, cu- 
yos rangos oscilaban entre +/- 1-2 metros. Sin embargo, en la última década, en la que se han venido manejando proyecciones inferiores al rango de 1 metro de subida, y sobre todo, en la que se ha mejorado sustancialmente la precisión de los modelos de cambio del nivel medio del mar, estas discrepancias metodológicas resultan mucho más relevantes.

La aproximación más detallada realizada sobre esta cuestión clave fue hecha por Titus y Narayanan (1995), al proponer aislar los efectos locales en una serie de registros temporales de niveles del mar, estimando el comportamiento futuro a partir de las componentes global (aportada por un fenómeno eustático) y local (estimada a partir de la extrapolación de las tendencias locales observadas). El principal obstáculo del método planteado por Titus y Narayanan (1995) estriba en que se requiere el uso de series de larga duración, de más de 100 años, para poder eliminar las tendencias locales de las proyecciones de futuro. Esta exigencia convierte esta aproximación en un método de uso tan restrictivo, que en la práctica resulta imposible aplicarlo en la mayor parte de los mareógrafos del mundo (como todos los de España, por ejemplo, ya que no disponen de series tan largas).

El objetivo de este trabajo consiste en calcular las expectativas de cambio del nivel medio del mar a finales del siglo XXI en las Islas Canarias, donde únicamente existen series temporales de corta duración, y donde por tanto no es aplicable la propuesta de Titus y Narayan (1995). Para ello se emplearán tanto los registros de los mareógrafos anclados en los principales puertos canarios como los de los satélites altimétricos que han obtenido datos en su entorno desde 1992, intentando, mediante el uso de estos datos, superar la escasa duración de las series temporales de los mareógrafos del archipiélago. Se enmarcaría, por tanto, dentro de la tercera aproximación expuesta anteriormente, considerándose que los registros de cambio del nivel del mar presentan una componente local y otra global, que de acuerdo con la mayor parte de la literatura científica sobre esta cuestión se verá acelerada en el futuro.

\section{2. Área de estudio}

El área de estudio elegida son las Islas Canarias (figura 1). Esta elección se justifica por los siguientes motivos: 1) la existencia de series temporales de mareógrafos y satélites altimétricos de suficiente longitud y con solape temporal que permiten testar la metodología desarrollada para este trabajo; 2) el profundo conocimiento existente en la bibliografía de referencia sobre la geología de las Islas Canarias, que permite una información más precisa que en otros territorios sobre la neotectónica de las áreas en las que están anclados los mareógrafos; y 3) la ausencia de estudios de este tipo en Canarias, frente a aproximaciones a esta cuestión realizadas tanto para todo el conjunto de la Península Ibérica (Tel y García, 2002), como centradas en diferentes regiones como el norte de España (Marcos et al., 2005, o las costas andaluzas (Fraile, 2011). 


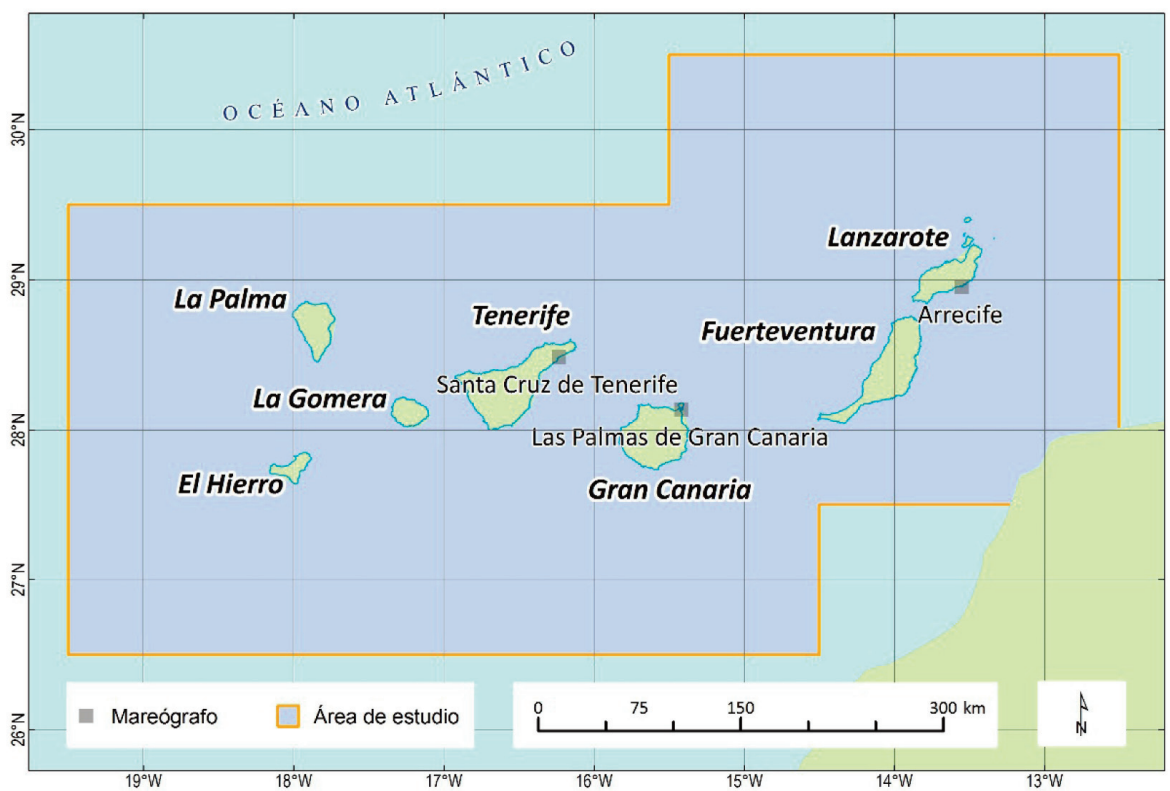

Figura 1. Área de estudio.

Las Islas Canarias son un archipiélago volcánico formado a partir de un punto caliente, de acuerdo con la teoría de mayor aceptación en la actualidad (Holik et al., 1991, Carracedo et al., 1998). La edad de cada una de las islas varía entre los 20 MA de Lanzarote, en el extremo NE y los 1.8 MA de El Hierro, al SW. La formación de cada una de las islas es un proceso complejo compuesto por varias fases de magmatismo (Meléndez Hevia, 2004).

Desde el punto de vista de los movimientos verticales de la superficie emergida que puedan afectar tanto a los registros recientes de los mareógrafos como, especialmente, a las potenciales inundaciones futuras, Meco et al. (2002) identificaron tasas de neotectónica inferiores a $0.1 \mathrm{~mm}$ año en el norte de Gran Canaria. De acuerdo con este modelo más aceptado, las islas centrales se encontrarían en un período de estabilidad tectónica (Rodríguez-González et al., 2009), mientras que las más occidentales (principalmente El Hierro) están aún experimentando un ligero ascenso tectónico, además de fenómenos de magmatismo reciente (Pérez-Torrado et al., 2012, Rodríguez-González et al., 2012). En las islas orientales se observa un fenómeno de ligera subsidencia, al tratarse de islas más antiguas (Carracedo et al., 2002). No obstante, los rangos de velocidades de cambio vertical de cada una de las islas resultan mínimos en comparación con las tasas de cambio del nivel medio del mar observadas en los océanos (Nichols et al., 2011) durante las últimas décadas, por lo que para los propósitos de este trabajo resultan despreciables. 


\section{Datos}

Para el desarrollo de este trabajo se emplearon tres tipos de datos:

- Registros medios anuales de los niveles del mar de los mareógrafos situados en las Islas Canarias, procedentes del Permanent Service for Mean Sea Level (PSMSL), con sede en Southampton (Reino Unido), organismo que recopila las serie temporales de los mareógrafos del planeta, a partir de los registros de diferentes instituciones nacionales. Dado que los mapas topográficos de cada isla emplean un datum topográfico (nivel de referencia o valor 0) local y propio, existe una red de mareógrafos de más densidad que en el resto del territorio español. Sin embargo, a excepción de los mareógrafos de Santa Cruz de Tenerife y Arrecife (Lanzarote), el resto de las series son cortas (solo desde la década de los 90) y, en ocasiones, además, presentan numerosas lagunas. Por esta razón solamente se emplearon los registros de los mareógrafos de Las Palmas de Gran Canaria (desde 1992 a la actualidad, pero con escasas lagunas), Lanzarote - Arrecife (desde 1955) y Santa Cruz de Tenerife (desde 1927). En este caso, los datos han sido tomados por el Instituto Españo de Oceanografía en los mareógrafos de Tenerife y Arrecife, y por Puertos del Estado en el caso de Las Palmas de Gran Canaria.

- Imágenes de altimetría por satélite procedentes de los satélites Topex / Poseidon, Jason-1 y Jason-2, pertenecientes a la Agencia Espacial Europea (ESA). Estas imágenes, de libre uso, se han obtenido ya tratadas por el CU Sea Level Research Group de la Universidad de Colorado (Estados Unidos), que proporciona una serie temporal semanal con inicio en julio de 1992 y está permanentemente actualizada. La información así obtenida tiene una resolución espacial de 1ํㅡ, y proporciona datos sobre la altura media de cada celdilla de $1^{\circ}$ medida respecto a la superficie del geoide, presentando una precisión vertical de hasta $0,15 \mathrm{~mm}$ (Ablain et al., 2009). La periodicidad de los datos adquiridos por estos sistemas de teledetección es semanal (Degnan, 1993), aunque para este trabajo se calcularon los promedios anuales, con el objetivo de equiparar la periodicidad de los registros a las de las otras fuentes de información.

- Serie temporal del nivel medio del mar a nivel global del CSIRO, elaborada por Church y White (2011), a partir de los registros de 250 mareógrafos de todo el planeta, a los que se ha filtrado la componente tectónica e isostática. La serie comienza en el año 1880 y termina en 2009 (figura 2), proporcionando un valor único anual para todo el planeta. 


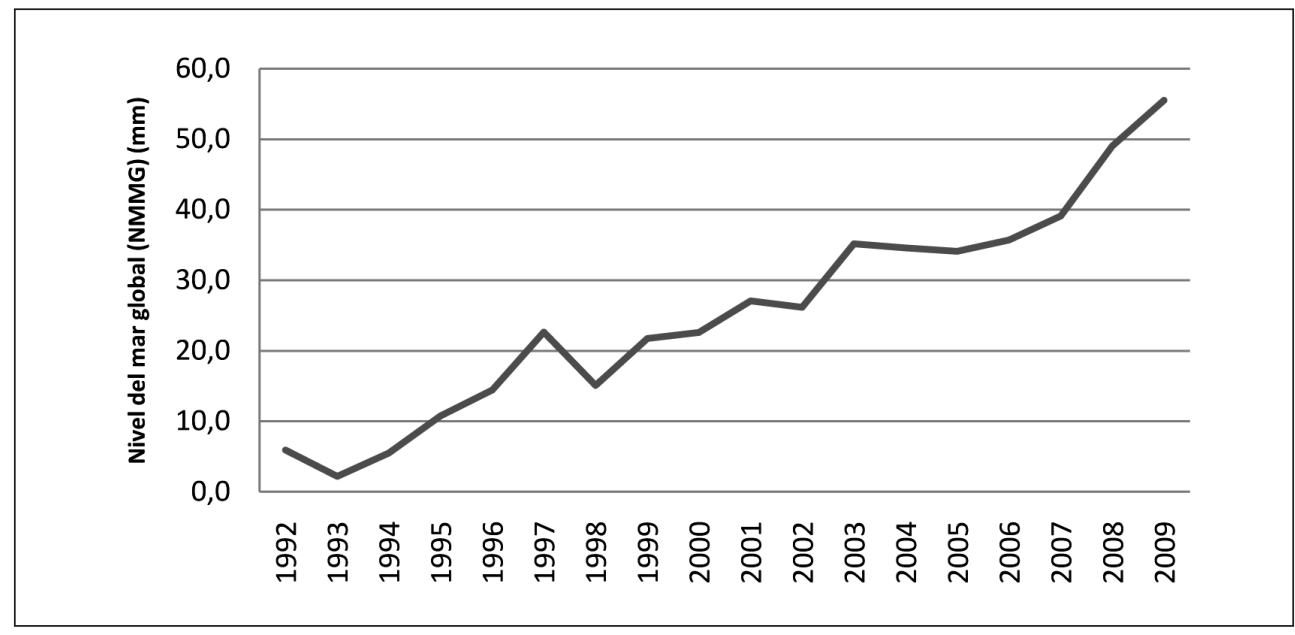

Figura 2. Serie temporal del nivel medio del mar global en el periodo 1992-2009.

Fuente: modificado de Church y White, 2011.

\section{Métodos}

El método desarrollado está basado en estimar las expectativas locales de cambio del nivel del mar en las Islas Canarias mediante la extrapolación hacia el futuro de la relación entre los registros recientes de niveles del mar y una serie temporal global integrada por registros de todos los mareógrafos del planeta. La relación reciente entre las series temporales "nivel medio del mar global" (NMMG, representada por la serie del CSIRO) y cada una de las series de "nivel medio del mar local" (NMML, representadas tanto por las series de los mareógrafos como por las de los satélites altimétricos) fue estimada mediante un modelo de regresión lineal simple, en el que la serie NMMG se consideró como variable independiente y la local NMML como dependiente (figura 3).

Sin embargo, realizar predicciones sobre subida del nivel del mar exige considerar que, de acuerdo con la bibliografía de referencia (Ding et al., 2001, IPCC, 2007, Stocker et al., 2013, Rahmstorf, 2007, Pfeffer et al., 2008), este fenómeno se acelerará significativamente en el futuro por encima de las tendencias registradas hasta el presente. De acuerdo con Titus y Narayanan (1995), la variable NMML, de carácter exclusivamente local, permanecerá estable, mientras que solamente la componente global se acelerará.

Por tanto, para poder predecir localmente los cambios futuros se deben utilizar tanto las tendencias locales recientes observadas por satélites y mareógrafos, como las estimaciones globales futuras de cambio del nivel medio del mar, que vienen proporcionadas por complejos modelos de cambio global elaborados por diferentes instituciones. 


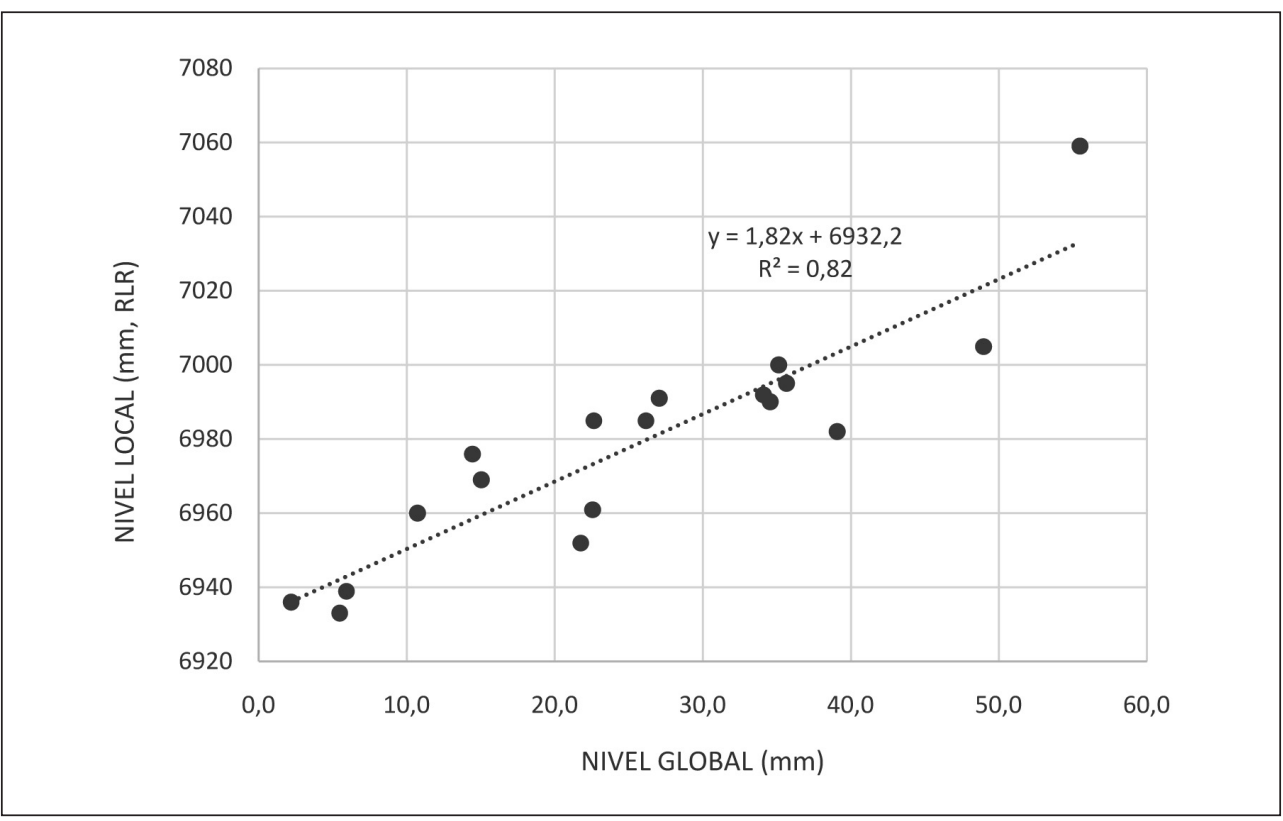

Figura 3. Diagrama de dispersión entre la serie global anual NMMG (Church y White, 2011 y la serie de registros medios anuales del mareógrafo de Las Palmas de Gran Canaria (NMML).

Asumiendo que la relación entre NMML y NMMG se mantendrá estable en el futuro, es posible realizar la predicción identificando el valor futuro de NMML a partir de la recta de regresión de x sobre y (en el diagrama de dispersión el valor de NMML correspondiente al valor esperado de NMMG), tomando como valor de la variable independiente NMMG la predicción realizada por un modelo global de cambio del nivel del mar en el futuro, que conlleva la aceleración global señalada por la mayor parte de la literatura científica sobre este tema.

En este trabajo se han empleado tres escenarios del IPCC para el período 2081-2100 como referencias futuras de NMMG: los escenarios RCP2.6, RCP4.5 y RCP8.5, siglas de la expresión inglesa Representative Concentration Pathway, sucedidas de un número que alude al forzamiento radiativo alcanzado por cada uno de ellos en el año 2100, expresado en $\mathrm{W}^{*} \mathrm{~m}^{-2}$. De todos modos, el método propuesto admite cualquier otro tipo de proyección global (Rahmstorff, 2007, Pfeffer et al., 2008), así como cualquier horizonte temporal futuro para el cual se hayan realizado predicciones globales.

Se calculó, por tanto, una recta de regresión lineal para cada serie temporal, tanto de mareógrafos como de altímetros embarcados en satélites, resultando finalmente 25 ecuaciones de rectas de regresión de x sobre y (3 rectas para los 3 mareógrafos, y 22 
rectas para las 22 celdillas de $1^{\circ} \times 1^{\circ}$ analizadas). Se obtuvieron 3 predicciones (valores de NMML sobre la recta de regresión) a partir de cada una de ellas (figura 4), correspondientes a los valores futuros de NMMG de $40 \mathrm{~cm}$ (RCP2.6), $46 \mathrm{~cm}$ (RCP4.5) y 63 cm (RCP8.5). El valor de subida neta se estimó a partir de la diferencia entre el valor de NMML predicho por el modelo y el valor medio de NMML durante el período 19922000 (Fraile et al., 2014).

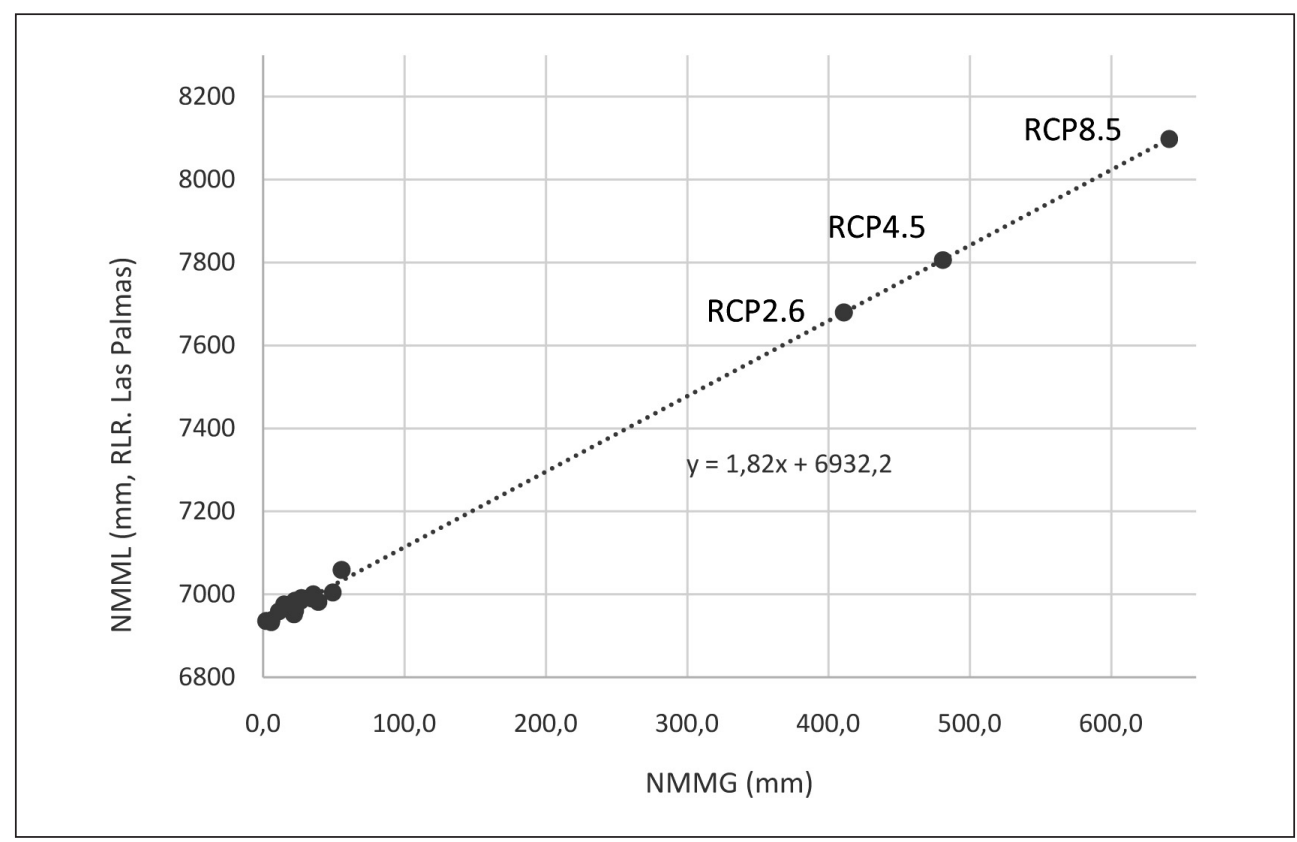

Figura 4. Estimación de niveles medios del mar futuros a partir de la relación entre una serie global (NMMG), una serie local (NMML), y escenarios globales (RCP2.6, RCP4.5 y RCP8.5), para el mareógrafo de Las Palmas de Gran Canaria.

La elección de una serie de registros medios anuales de cada serie de NMML y NMMG, en lugar de emplear registros medios mensuales (que podrían haber aportado una mayor cantidad de información en la relación entre ambas variables, al haber más parejas de datos) se explica por presentar ambas series estacionalidades muy dispares: en el caso de la serie NMMG la estacionalidad es muy difusa o nula, al estar integrada por registros de mareógrafos tanto del hemisferio norte como del hemisferio sur, mientras que cualquier serie NMML presenta una clara estacionalidad intraanual, con mínimos en junio y máximos claros en octubre (en el hemisferio norte). Por lo tanto, cualquier análisis de correlación responde mejor en este caso a una serie de periodicidad anual que a una serie de periodicidad mensual, pese a lo reducido del número de registros. 
Un aspecto crítico a la hora de realizar estimaciones de subida del nivel del mar es el cálculo de errores, tal y como ha sido subrayado en diversas publicaciones (Titus y Anderson, 2009, Little et al., 2013). En este caso se estimaron los márgenes de confianza de cada cálculo de cambio local del nivel del mar como resultado de la integración (por suma) de dos elementos: i) los márgenes de confianza de cada escenario del IPCC utilizados, tal y como han sido publicados por el IPCC (IPCC, 2013) con un nivel de confianza del 95\% (tabla 1), y ii) la estimación de la incertidumbre de los residuos del análisis de la regresión (con el mismo nivel de confianza) entre la serie temporal local y la del CSIRO, una vez que fue comprobada la normalidad y la homocedasticidad de dichos residuos mediante el test de Kolmogorov-Smirnoff (Chakravarti et al., 1967).

Tabla 1. Subidas del nivel medio del mar globales esperadas por modelo y escenario, y error asociado al nivel de confianza del $95 \%$ en cada uno de los escenarios en el periodo 2081-2100 (IPCC, 2013)

\begin{tabular}{|l|c|c|}
\hline Escenario & Incremento $(\mathrm{cm})$ & Error $(\mathrm{cm})$ \\
\hline RCP2.6 & 40 & 14,5 \\
RCP6.0 & 47 & 15 \\
RCP8.5 & 63 & 19 \\
\hline
\end{tabular}

Un aspecto clave del método propuesto es que es preciso asumir que es posible estimar valores fuera de la nube de puntos utilizados para el cálculo de la línea de regresión (ver el ejemplo de los registros del mareógrafo de Las Palmas, en la figura 4). Si bien los autores de este artículo son conscientes de que no son las condiciones deseables para realizar predicciones (Montgomery et al., 2012), se realiza esta asunción al no existir datos intermedios entre la nube de puntos y el valor de la variable independiente (es decir, las variables NMML y NMMG aún no han tomado valores entre el presente y el final del siglo XXI) que permitan realizar un análisis más preciso.

\section{Resultados}

Los resultados obtenidos son expectativas locales de cambio del nivel medio del mar correspondientes a cada uno de los tres escenarios elegidos para el análisis, para cada uno de los tres mareógrafos analizados y para cada celdilla de $1^{\circ} \mathrm{x} 1^{\mathrm{O}}$ de las aguas de las Islas Canarias. Aparecen sintetizados en la tabla 2 y en las figuras 5, 6 y 7. 
Tabla 2. Expectativas de cambio del nivel medio del mar (en $\mathrm{cm}$ ) en el período 2081-2100 en los mareógrafos analizados

\begin{tabular}{|l|c|c|c|c|}
\hline $\begin{array}{c}\text { Escenario de subida } \\
\text { del nivel del mar }\end{array}$ & $\begin{array}{c}\text { Umbrales de } \\
\text { probabilidad }\end{array}$ & $\begin{array}{c}\text { Santa Cruz de } \\
\text { Tenerife }\end{array}$ & $\begin{array}{c}\text { Las Palmas de } \\
\text { Gran Canaria }\end{array}$ & Arrecife \\
\hline \multirow{3}{*}{ RCP2.6 $(+40 \mathrm{~cm})$} & $<2,5 \%$ & 64,9 & 52,8 & 0,1 \\
& Media & 82,5 & 70,3 & 18,6 \\
& $>97,5 \%$ & 100,1 & 87,8 & 35,1 \\
\hline \multirow{3}{*}{ RCP4.5 $(+47 \mathrm{~cm})$} & $<2,5 \%$ & 79,3 & 65 & 3,1 \\
& Media & 97,4 & 83 & 22,1 \\
& $>97,5 \%$ & 115,5 & 101 & 41,1 \\
\hline \multirow{3}{*}{ RCP8.5 $(+63 \mathrm{~cm})$} & $<2,5 \%$ & 109,4 & 90,2 & 7,1 \\
& Media & 131,5 & 112,2 & 30,1 \\
& $>97,5 \%$ & 153,6 & 134,2 & 53,1 \\
\hline
\end{tabular}

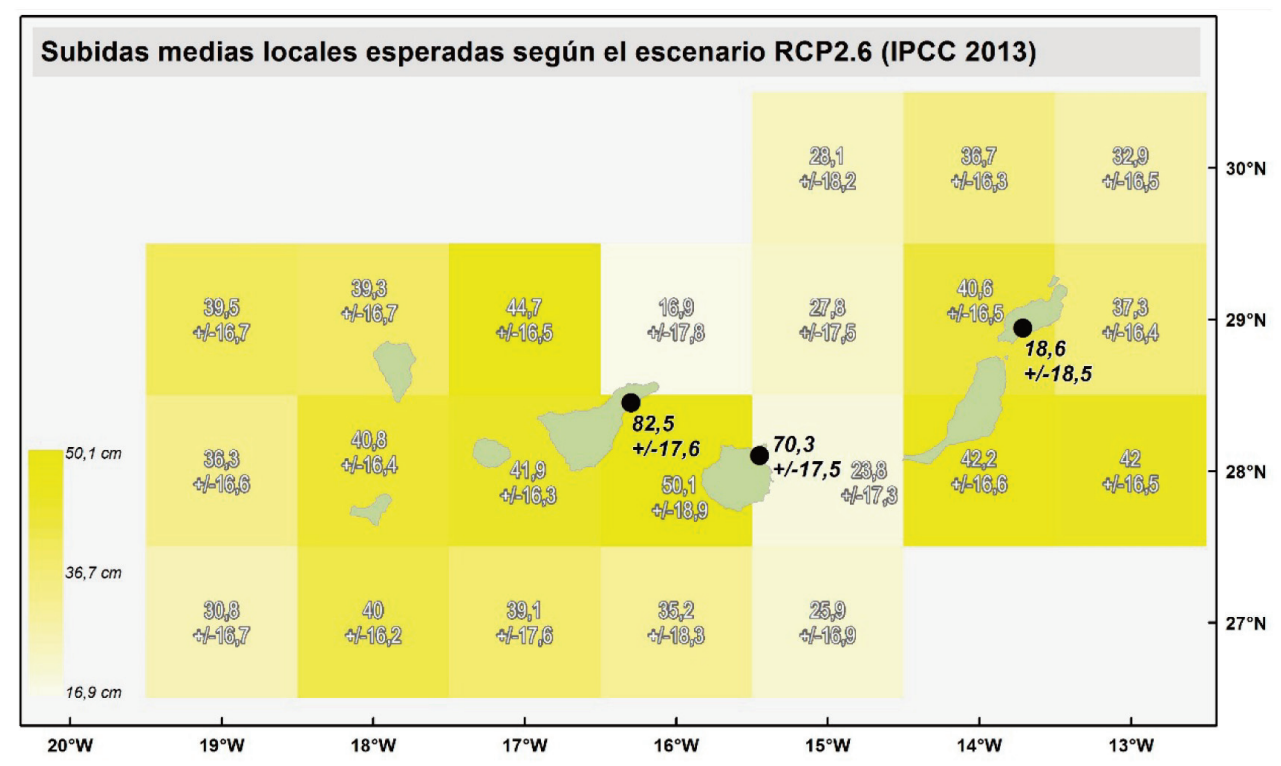

Figura 5. Expectativas de subida del nivel medio del mar en Islas Canarias en el período 2081-2100 (en cm) según el escenario RCP2.5 del IPCC (2013), y error asociado a cada celdilla para un nivel de confianza del $95 \%$. 


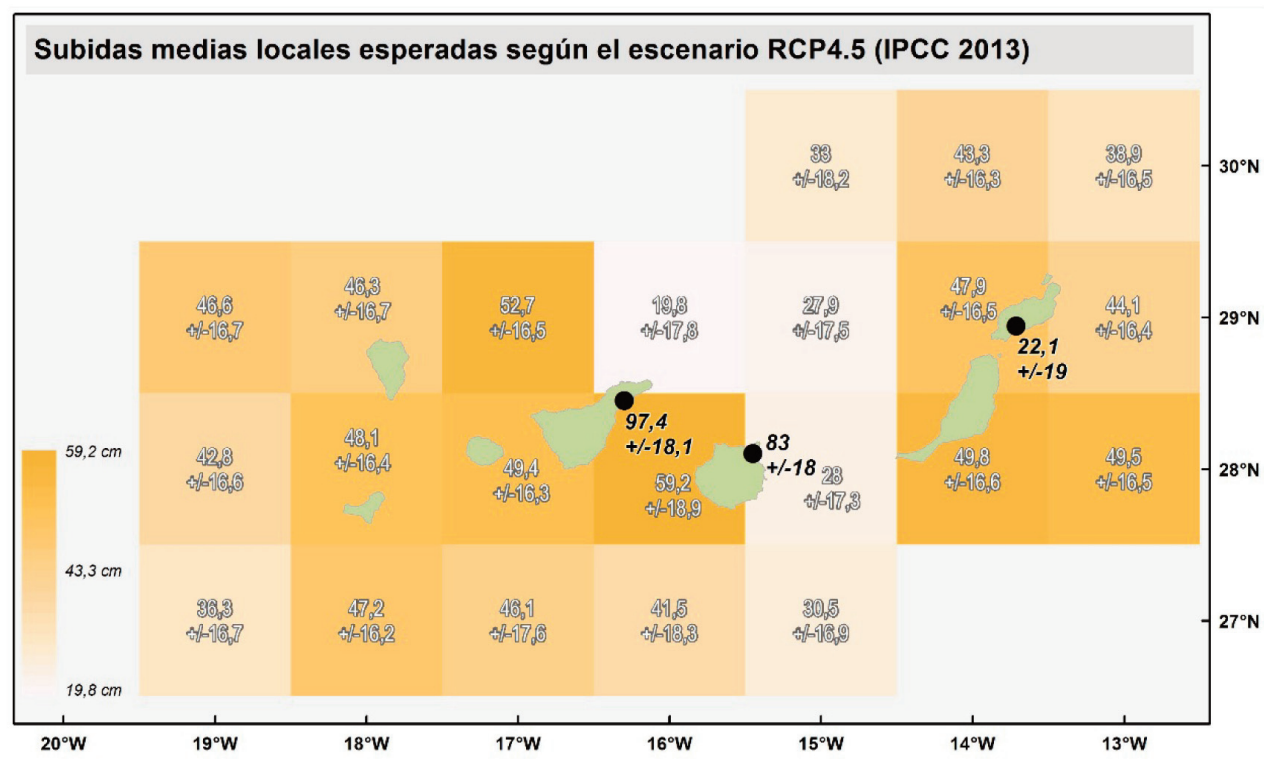

Figura 6. Expectativas de subida del nivel medio del mar en Islas Canarias en el período 2081-2100 (en cm) según el escenario RCP4.5 del IPCC (2013), y error asociado a cada celdilla para un nivel de confianza del 95\%.

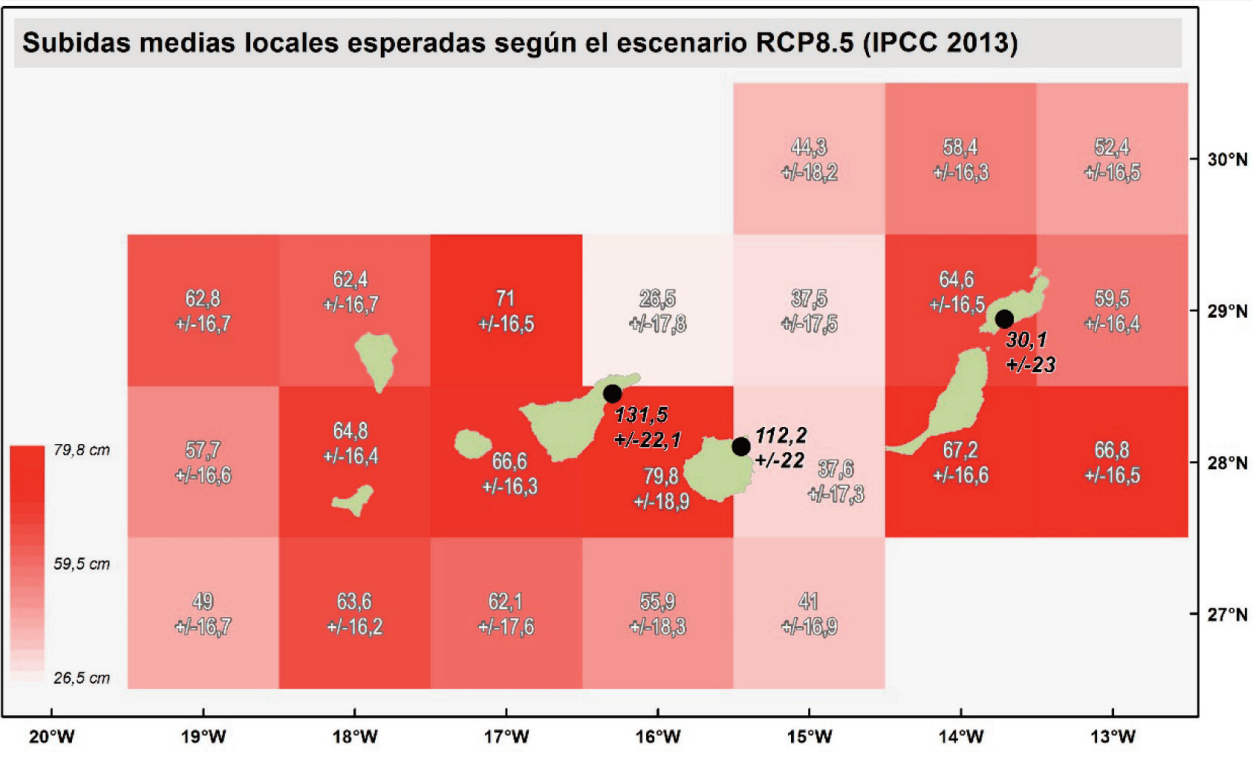

Figura 7. Expectativas de subida del nivel medio del mar en Islas Canarias en el período 2081-2100 (en cm) según el escenario RCP8.5 del IPCC (2013), y error asociado a cada celdilla para un nivel de confianza del $95 \%$. 
La tabla 2 muestra las expectativas de cambio del nivel medio del mar a finales del siglo XXI estimadas a partir de datos de los mareógrafos de Santa Cruz de Tenerife, Las Palmas y Arrecife, para dichas localizaciones. Los resultados obtenidos a partir de los registros de Santa Cruz de Tenerife y Las Palmas de Gran Canaria indican que el nivel del mar subirá por encima de cualquiera de los escenarios del IPCC (2013), independientemente del umbral de probabilidad tomado (bajo, medio o alto) a finales del siglo XXI. El mareógrafo de Arrecife, por el contrario, muestra valores muy inferiores a las expectativas del IPCC, incluso considerando el umbral alto $(97,5 \%)$ de probabilidad.

Las estimaciones calculadas a partir de los registros de los altímetros embarcados en satélites (figuras 5, 6 y 7) para las aguas de las Islas Canarias muestran valores muy semejantes a los predichos por el IPCC, salvo en el caso de las celdillas situadas en torno a $15^{\circ}$ y $16^{\circ} \mathrm{W}$, donde las expectativas obtenidas de cambio del nivel medio del mar son muy inferiores.

\section{Discusión}

Los resultados obtenidos en las aguas canarias, procedentes exclusivamente del análisis de los registros de satélites altimétricos, muestran un notable grado de coherencia interna, con valores muy parejos a los predichos por el modelo del IPCC en la mayor parte del área de estudio, y subidas muy inferiores en las aguas al norte y al este de Gran Canaria y al oeste de Fuerteventura. El descenso observado en este sector a partir del año 2006, siguiendo una tendencia opuesta a la de la curva global del nivel medio del mar, parece explicar estas moderadas expectativas de cambio futuro en este sector, sin que se haya encontrado en la literatura científica una explicación a este fenómeno, ni por tanto, a las predicciones realizadas.

Los resultados obtenidos a partir de los datos de los mareógrafos tienen una interpretación notablemente más compleja, puesto que en sus series temporales se reflejan, esencialmente, dos tipos de factores: los marinos (que son los únicos presentes en las series temporales de los altímetros embarcados en satélites) y los de la superficie emergida (vinculados generalmente a movimientos tectónicos y/o neotectónicos). Las diferencias observadas en la tabla 2 entre las predicciones globales del IPCC y los resultados de este trabajo para los mareógrafos de Las Palmas de Gran Canaria y Santa Cruz de Tenerife podrían sugerir algún fenómeno de ligera subsidencia local, puesto que en las aguas de su entorno no se han identificado expectativas de cambio coherentes con estos resultados. La principal explicación, por tanto, resulta ser de origen neotectónico. Este fenómeno ya ha sido identificado en la literatura científica en diversos sectores del planeta, con subidas aceleradas del nivel del mar en sectores costeros que duplican y triplican los valores de las aguas marinas (Sallenger et al., 2012) e incluso, debido a procesos isostáticos, el fenómeno inverso, con descensos del nivel del mar en registros de mareógrafos frente a ascensos de las aguas marinas (Johansson et al., 2004). 
No obstante, y en un contexto estructural y geológico regional, este último aspecto ha sido negado por Meco et al. (2002), que no solo no identifican ningún fenómeno de descenso de la superficie emergida en el sector septentrional de Gran Canaria, donde se encuentra localizado el mareógrafo estudiado, sino que en todo caso han registrado ligeras tasas de ascenso neotectónico inferiores a $+0,2 \mathrm{~mm} /$ año. De haberse mantenido durante las dos últimas décadas la tendencia tectónica observada desde el Pleistoceno por estos autores, la explicación de las discrepancias observadas entre mareógrafo y su entorno marino debería residir en movimientos locales de la superficie emergida, ya sea por causas neotectónicas, por cambios indebidos en la ubicación del instrumento, o por fenómenos de hundimiento de las infraestructuras sobre las que estos se asientan, tal y como ha sido reflejado en las campañas de recalibración de nivelación de mareógrafos en las que se han observado diferencias de hasta $20 \mathrm{~cm}$ con respecto al valor inicial, en periodos inferiores a dos décadas (Emery et al., 1988).

El caso de Arrecife resulta opuesto al de los otros dos mareógrafos, puesto que las expectativas de cambio del nivel medio del mar obtenidas se encuentran muy por debajo de las expectativas globales obtenidas por los modelos del IPCC empleados. Al igual que en el caso anterior, resulta difícil identificar las causas de este descenso. De acuerdo con la literatura científica sobre esta isla, cabría esperar una ligera subsidencia, al encontrarse en el sector oriental de Canarias, más antiguo (Meléndez Hevia, 2004). No obstante, los resultados indican que el fenómeno que parece producirse es el inverso, especialmente al comparar las expectativas locales obtenidas con las de las celdillas contiguas a Fuerteventura, cuyos valores son muy superiores. La determinación de las causas de este fenómeno queda fuera de los métodos de análisis empleados en este trabajo, exigiendo tanto de un proceso de análisis neotectónico del mareógrafo en el contexto de la isla de Lanzarote, para identificar los límites espaciales del aparente fenómeno de ascenso del mareógrafo, como de una revisión de la historia del instrumento, para identificar posibles cambios de posición.

Metodológicamente, se ha realizado una aproximación a la predicción de las expectativas de cambio local, que en publicaciones anteriores se han basado fundamentalmente i) en el uso de expectativas globales (Hallegate et al., 2011, Timmerman et al., 2010), ii) en aproximaciones cuaternaristas a los análisis de antiguos niveles del mar (Lambeck et al., 2011), o iii) en menor medida, en la estimación y comparación de tasas globales con las registradas localmente (Titus y Narayanan, 1995, Woodworth, 1999, Fraile y Ojeda, 2012, Sallenger et al., 2012), aunque empleando métodos de mayor exigencia en los parámetros de los datos de entrada para realizar el análisis estadístico.

Aunque es evidente que cuanto mayor sea el número de registros, mayor será la calidad y precisión del análisis realizado, es fundamental destacar la independencia del método desarrollado frente a la escasa continuidad espacio temporal de las series, lo que acaba por convertirse en una limitación notable frente a la mayoría de métodos estadísticos alternativos, como la comparación de las tendencias locales y globales (Ti- 
tus y Narayanan, 1995), el análisis de componentes armónicos aplicados a este problema (Pugh, 1986 o las funciones empíricas ortogonales (Marcos et al., 2005) de gran potencia analítica pero de estrictas exigencias en los datos de entrada. De este modo se abren nuevas posibilidades analíticas en series temporales frecuentemente ignoradas debido a las fuertes exigencias de continuidad temporal por parte de otros métodos.

Por otra parte, y si bien diferentes autores han recalcado los riesgos de realizar predicciones fuera de la nube de puntos (Montogomery et al., 2012) -tal y como se ha planteado en este trabajo- la estimación de los errores (Titus, 2009) una vez comprobada su homocedasticidad, parece paliar los problemas asociados a este tipo de asunciones.

\section{Conclusiones}

El método empleado demuestra ser eficiente en la medida en que permite evaluar cambios del nivel medio del mar en el futuro a partir de la comparación entre dos series temporales relativamente cortas de datos de mareógrafos y/o altímetros embarcados en satélites.

Las ventajas del uso de este método consisten en: i) obtener una ecuación sencilla que permite realizar predicciones sobre el cambio local del nivel medio del mar a partir de cualquier modelo global de cambio del nivel del mar, y ii) poder realizar predicciones con márgenes de confianza bastante precisos, si los residuos del análisis de regresión se comportan de manera normal y homocedástica. En este sentido, resulta crítico recalcar que los errores, tanto los procedentes de los modelos globales externos, como los inherentes al análisis de regresión realizado, deben ser considerados en cualquier publicación científica que aborde la cuestión de las predicciones de subida del nivel del mar.

En cuanto a los resultados obtenidos, es preciso subrayar que, dentro de un contexto generalizado de subida del nivel medio del mar (a escala global y en el área de estudio), se observa una amplia variabilidad espacial en las predicciones, especialmente en el caso de las predicciones hechas a partir de los datos procedentes de los mareógrafos, que es concordante con las publicaciones al respecto. En el caso de las predicciones basadas en los datos procedentes de los altímetros embarcados en satélites, los resultados obtenidos indican una notable semejanza con las expectativas globales publicadas, aunque la escasa duración del solape entre los registros de los datos altimétricos y de la serie global empleada (17 años) exigirá realizar actualizaciones de este análisis cuando nuevos registros estén disponibles. 


\section{Agradecimientos}

Los autores agradecen los comentarios y sugerencias realizados por Enrique Arias Ibáñez, así como el apoyo proporcionado por el Departamento de Geografía de la Universidad de Las Palmas de Gran Canaria, dentro del proyecto Diagnóstico ambiental de los sistemas de dunas de Canarias para la elaboración de modelos sostenibles de gestión territorial (CSO2010-18150) financiado por el Ministerio de Ciencia e Innovación y por Fondos Feder. Asimismo, parte de esta investigación se ha desarrollado en la Florida International University, mediante una estancia postdoctoral realizada con fondos del Vicerrectorado de Investigación de la Universidad de Sevilla.

\section{Bibliografía}

Ablain, M., Cazenave, A., Valladeau, G., y Guinehut, S., 2009. A new assessment of the error budget of global mean sea level rate estimated by satellite altimetry over 1993-2008. Ocean Science, 5(2), 193-201.

Bruun, P., 1954. Migrating sand waves or sand humps, with special reference to investigations carried out on the Danish North Sea Coast. Coastal Engineering Proceedings, vol. 1 (5), 21.

Camino, E.R., Ruggeroni, J.R.P., y Hernández, F.H., 2014. Quinto informe de evaluación del IPCC: Bases físicas. Tiempo y Clima, 11(1).

Carracedo, J.C., Day, S., Guillou, H., Badiola, E.R., Canas, J.A., y Perez-Torrado, F.J., 1998. Hotspot volcanism close to a passive continental margin: the Canary Islands. Geological Magazine 135, 591-604.

Carracedo, J.C., Pérez Torrado, F.J., Ancochea, E., Meco, J., Hernán, F., Cubas, C.R. y Ahijado, A., 2002. Cenozoic volcanism II: the Canary Islands. Londres: The Geological Society.

Chakravarti, Laha, y Roy, 1967. Handbook of Methods of Applied Statistics, Volume I. John Wiley and Sons, 392-394.

Church, J.A. y White, N.J., 2011. Sea-level rise from the late 19th to the early 21st century. Surveys in Geophysics, vol. 32 (4-5), 585-602.

Dean, R.G., 1991. Equilibrium beach profiles: characteristics and applications. Journal of Coastal Research, 7 (1), 53-84.

Dean, R.G., Dalrymple R., Faribridge, R., Leatherman, S., Nummeda, D., O'Brien, M., Piley, O., Sturges W. y Wiegel, R., 1987. Responding to sea level changes: engineering implications, Washington: National Academy Press.

Degnan, J.J., 1993. Millimeter accuracy satellite laser ranging: a review. Contributions of space geodesy to geodynamics: technology, p. 133-162.

Ding, Y.D.J.G., Griggs, D.J., Noguer, M., van der Linden, P.J., Dai, X., Maskell, K., y Johnson, C.A., 2001. Climate change 2001: the scientific basis. Cambridge. Cambridge University Press.

Douglas, B., Kearney, M.T., y Leatherman, S.P., 2000. Sea level rise: History and consequences. San Diego. Academic Press. 
Emery, K.O., Aubrey, D.G., y Goldsmith, V., 1988. Coastal neo-tectonics of the Mediterranean from tide-gauge records. Marine Geology, 81(1), 41-52.

Fraile Jurado, P., 2011. Análisis de las problemáticas asociadas a la espacialización, evolución y representación de niveles del mar presentes y futuros en Andalucía. Sevilla. Universidad de Sevilla.

Fraile Jurado, P. y Ojeda Zújar, J., 2012. Evaluación de la peligrosidad asociada al aumento de la superficie inundable por la subida del nivel medio del mar en la costa entre Cádiz y Tarifa. Geofocus. Revista Internacional de Ciencia y Tecnología de la Información Geográfica, 12, 329-348.

Fraile Jurado, P., Sánchez Carnero y Ojeda Zújar, J., 2014. Sensibilidad del cálculo de los niveles medios del mar al método y periodo de las series temporales de los mareógrafos en los procesos de inundación: Valdelagrana (Cádiz). Boletín de la Asociación de Geógrafos Españoles, 65, 59-70.

Gornitz, V., Lebedeff, S. y Hansen, J., 1982. Global sea level trend in the past century. Science, 215 (4540), 1611-1614.

Hallegatte, S., Ranger, N., Mestre, O., Dumas, P., Corfee-Morlot, J., Herweijer, C. y Wood, R.M., 2011. Assessing climate change impacts, sea level rise and storm surge risk in port cities: a case study on Copenhagen. Climatic change, 104 (1), 113-137.

Hoffman, J.S., Keyes, D.L. y Titus, J.G., 1983. Projecting future sea level rise: methodology, estimates to the year 2100, and research needs. Strategic Studies Staff, Office of Policy Analysis, Office of Policy and Resource Management, US Environmental Protection Agency.

Holik, J.S., Rabinowitz, P.D. y Austin, J.A., 1991. Effects of Canary Hotspot Volcanism on Structure of Oceanic-Crust Off Morocco. Journal of Geophysical Research-Solid Earth and Planets 96, 12039-12067.

IPCC, 2007. Climate Change 2007: The Physical Science Basis. Contribution of Working Group I to the Fourth Assessment Report of the Intergovernmental Panel on Climate Change [Solomon, S., D. Qin, M. Manning, Z. Chen, M. Marquis, K.B. Averyt, M. Tignor and H.L. Miller (eds.)]. Cambridge University Press, Cambridge, United Kingdom and New York, NY, USA, 996 pp.

IPCC, 2013. Climate Change 2013: The Physical Science Basis. Contribution of Working Group I to the Fifth Assessment Report of the Intergovernmental Panel on Climate Change [Stocker, T.F., D. Qin, G.-K. Plattner, M. Tignor, S.K. Allen, J. Boschung, A. Nauels, Y. Xia, V. Bex and P.M. Midgley (eds.)]. Cambridge University Press, Cambridge, United Kingdom and New York, NY, USA, 1535 pp.

Johansson, M.M., Kahma, K.K., Boman, H. y Launiainen, J., 2004. Scenarios for sea level on the Finnish coast. Boreal Environment Research, 9 (2), 153-166.

Lambeck, K., Antonioli, F., Anzidei, M., Ferranti, L., Leoni, G., Scicchitano, G. y Silenzi, S., 2011. Sea level change along the Italian coast during the Holocene and projections for the future. Quaternary International, 232(1), 250-257.

Little, C.M., Urban, N.M. y Oppenheimer, M., 2013. Probabilistic framework for assessing the ice sheet contribution to sea level change. Proceedings of the National Academy of Sciences, 110 (9), 3264-3269. 
Marcos, M., Gomis, D., Monserrat, S., Álvarez Fanjul, E., Pérez, B. y García Lafuente, J., 2005. Consistency of long sea level time series in the northern coast of Spain. Journal of Geophysical Research: Oceans. 110. C03008, doi:10.1029/2004JC002522.

Meco, J., Guillou, H., Carracedo, J.C., Lomoschitz, A., Ramos, A.J.G. y Rodrıguez-Yánez, J.J., 2002. The maximum warmings of the Pleistocene world climate recorded in the Canary Islands. $\mathrm{Pa}$ laeogeography, palaeoclimatology, palaeoecology, 185 (1), 197-210.

Medina, R., Losada, I.J., Méndez, F.J., Olabarrieta, M., Liste, M., Menéndez, M. y Luceño, A., 2004. Impacto en la costa española por efecto del cambio climático. Oficina Española de Cambio Climático-Dirección General de Costas Ministerio de Medio Ambiente.

Meléndez Hevia, I., 2004. Geología de España: una historia de seiscientos millones de años. Madrid: Rueda.

Montgomery, D.C., Peck, E.A. y Vining, G., 2012. Introduction to linear regression analysis. Nueva York: John Wiley y Sons.

Moss, R.H., 2011. Reducing doubt about uncertainty: Guidance for IPCC's third assessment. Climatic change, 108 (4), 641-658.

Nicholls, R.J., Hanson, S.E., Lowe, J.A., Warrick, R.A., Lu, X., Long, A.J. y Carter, T.R., 2011. Constructing sea-level scenarios for impact and adaptation assessment of coastal areas: A guidance document. Supporting Material, Intergovernmental Panel on Climate Change Task Group on Data and Scenario Support for Impact and Climate Analysis (TGICA). Ginebra, IPCC.

Pardo, J.E., Verger, I. y Rosselló, V., 1991. La erosión antrópica en el litoral valenciano. Valencia: Generalitat Valenciana.

Pérez-Torrado, F.J., Carracedo, J.C., Rodríguez-González, A., Soler, V., Troll, V.R. y Wiesmaier, S., 2012. La erupción submarina de La Restinga en la isla de El Hierro, Canarias: Octubre 2011Marzo 2012. Estudios geológicos, 68.

Pfeffer, W.T, Harper, J.T. y O'Neel, S., 2008. Kinematic constraints on glacier contributions to 21stcentury sea-level rise. Science, 321 (5894), 1340-1343.

Puertos del Estado, 2009. REDMAR: Informe anual 2009. Madrid: Puertos del Estado.

Pugh, D.T., 1996. Tides, surges and mean sea-level (reprinted with corrections). Nueva York: John Wiley y Sons Ltd.

Rahmstorf, S., 2007. A semi-empirical approach to projecting future sea-level rise. Science, 315 (5810), 368-370.

Rice, R.J., Saldaña, M.P.V., Elorza, M.G. y Hevia, G.M., 1982. Fundamentos de geomorfología. Madrid: Thomson-Paraninfo.

Rodríguez-González, A., Fernández-Turiel, J.L., Pérez-Torrado, F.J., Hansen, A.R., Aulinas, M., Carracedo, J.C., Gimeno, D., Guillou, H., Paris, R. y Paterne, M., 2009. The Holocene volcanic history of Gran Canaria island: implications for volcanic hazards. Journal of Quaternary Science 24, 697-709.

Rodríguez-González, A., Pérez-Torrado, F.J., Fernández-Turiel, J.L., Carracedo, J.C. y Guillou, H., 2012. Modelado geomorfológico con técnicas SIG de erupciones volcánicas generadoras de plataformas costeras: el volcán de Montaña del Tesoro (El Hierro, Islas Canarias). Geo-Temas. DOI: http://hdl.handle.net/10261/53760 
Sallenger Jr, A.H., Doran, K.S. y Howd, P.A., 2012. Hotspot of accelerated sea-level rise on the Atlantic coast of North America. Nature Climate Change, 2 (12), 884-888.

Tel, E. y García, M.E., 2002. Nivel del mar en las costas españolas y su relación con el clima. Comunicación en III Congreso de la Asociación Española de Climatología. Palma de Mallorca. 101-110.

Timmermann, A., McGregor, S. y Jin, F., 2010. Wind effects on past and future regional sea level trends in the Southern Indo-Pacific. Journal of Climate, 23 (16), 4429-4437.

Titus J., 2009. Coastal sensitivity to sea level rise: a focus in the Mid-Atlantic Region. Washington: US Climate Change Program. (USCCP).

Titus, J.G. y Anderson, K.E., 2009. Coastal sensitivity to sea-level rise: A focus on the Mid-Atlantic region. Washington: Government Printing Office.

Titus, J.G. y Narayanan, V.K., 1995. The probability of sea level rise. Washington: US Environmental Protection Agency.

Titus, J.G. y Richman, C., 2001. Maps of lands vulnerable to sea level rise: modeled elevations along the US Atlantic and Gulf coasts. Climate Research,18(3), 205-228.

Woodworth, P. L., 1987. Trends in UK mean sea level. Marine Geodesy, 11 (1), 57-87.

Woodworth, P.L., 1999. High waters at Liverpool since 1768: the UK's longest sea level record. Geophysical Research Letters, 26 (11), 1589-1592.

Woodworth, P.L., Tsimplis, M.N., Flather, R.A. y Shennan, I., 1999. A review of the trends observed in British Isles mean sea level data measured by tide gauges. Geophysical Journal International, 136 (3), 651-670.

Zazo, C., Dabrio, C.J., Goy, J.L., Bardají, T., Ghaleb, B., Lario, J., Hoyos, M., Hillaire-Marcel, C., Sierro, F., Flores, J.A., Silva, P.G. y Borja, F., 1996. Cambios en la dinámica litoral y nivel del mar durante el Holoceno en el Sur de Iberia y Canarias Orientales. Geogaceta, 20 (7), 1679-1682.

Zazo, C., Mercier, N., Silva, P.G., Dabrio, C.J., Goy, J.L., Roquero, E. y de Luque, L., 2005. Landscape evolution and geodynamic controls in the Gulf of Cadiz (Huelva coast, SW Spain) during the Late Quaternary. Geomorphology, 68(3), 269-290. 